\title{
Avaliação do Nível de Exposição à Vibração de Operadores de Empilhadeiras
}

\author{
Cristiano Zuccol ${ }^{1}$,Herbert M. Gomes ${ }^{2}$
}

\section{Resumo}

Este trabalho apresenta um estudo de vibração de corpo inteiro em operadores de máquinas empilhadeiras, operando em diferentes situações, comparando dois tipos de pneus, super-elásticos (maciços) e pneumáticos, e em dois tipos de piso, paralelepípedo e asfalto liso, na condição de carregadas (com carga nos garfos) e descarregado (sem carga) sob velocidades equivalentes. A vibração foi medida nos três eixos ortogonais, $\mathrm{x}$, y e z e, a partir daí, calculada uma aceleração ponderada para comparação dos resultados com a norma ISO 2631-1. O objetivo principal desse trabalho é avaliar a vibração suportada, no corpo inteiro, pelos operadores das empilhadeiras, os quais estão expostos a essa ação repetitiva e possuem histórico de reclamações da agressão sofrida em suas colunas vertebrais durante a operação nesse tipo de máquina. Com os resultados obtidos, pôde-se perceber que, de um modo geral, o pneu do tipo pneumático apresentou valores menores para a aceleração equivalente. Neste caso, o pneu superelástico apresentou valores de amplitude de aceleração maiores, portanto, mais prejudicial à saúde do operador.

Palavras-Chave: Vibração em empilhadeiras, medição de aceleração de corpo inteiro, medição experimental.

\footnotetext{
${ }^{1}$ Eng. Mecânico, Linck S.A. Equipamentos Rodoviários e Industriais, Av. das Industrias, 500 - Km 106,5 da BR-290, CEP:92900-000, Eldorado do Sul, RS, Brazil. Tel.(+55 51) 2125-3362, Fax.: (+55 51) 21254545. e-mail: zucco@linckmaquinas.com.br

${ }^{2}$ Prof. Dr., UFRGS, Universidade Federal do Rio Grande do Sul, Programa de Pós Graduação em Engenharia Mecânica, Porto Alegre, Rio Grande do Sul, Brasil. Tel.: (+55 51) 3308-3681, email:herbert@mecanica.ufrgs.br
} 


\section{Introdução}

É usual distinguirem-se, essencialmente, duas categorias principais de vibrações: as que envolvem o corpo como um todo e as que envolvem apenas alguns dos respectivos segmentos, como por exemplo, o sistema mão-braço. As vibrações transmitidas ao corpo inteiro são geralmente de baixa freqüência e grande amplitude, situam-se na faixa de 1 a $80 \mathrm{~Hz}$, mais especificamente 1 a $20 \mathrm{~Hz}$. Também são enquadrados, como vibração no corpo inteiro, os casos de enjôo que compreendem as frequiências na faixa de 0,1 a $0,63 \mathrm{~Hz}$. Tais vibrações são mais críticas em atividades relacionadas aos meios de transporte. O maquinário com aplicação industrial ou rodoviário expõe o ser humano à vibração mecânica, interferindo diretamente no seu conforto, na eficiência do seu trabalho, na saúde e, em alguns casos, na segurança. Não obstante, existem outros fatores que, da mesma maneira, causam vibração. A má postura do operador, predisposição genética e, principalmente, a soma desses fatores, pode conduzir a problemas como traumatismos cervicais, abdominais e digestivos, dificuldades urinárias, desordem visual, dor de cabeça, insônia entre outros sintomas similares (Griffin, 2000).

Vibrações que atingem um segmento do corpo (localizadas) são estudadas com mais freqüência(ISO 5349 -1 e 2, 1997) e situam-se na faixa de 6,3 a $1250 \mathrm{~Hz}$. Ocorre, principalmente nos trabalhadores que envolvem atividades de escavação, na construção de galerias, quebra de pedras e madeireiras. Segundo estimativas de Donald Wasserman appud Vendrame (2003), há mais de dois milhões de trabalhadores expostos aos riscos das vibrações de mãos e braços nos Estados Unidos e este número é maior no resto do mundo. Muitos deles são usuários regulares de ferramentas manuais pneumáticas, elétricas, hidráulicas ou movidas a combustível. A avaliação do nível de exposição é realizada com base no cálculo da exposição diária expressa em aceleração ponderada para um período de 8 horas (jornada de trabalho).

Neste trabalho, os movimentos transmitidos ao corpo humano, como um todo, se dão através do assento de uma empilhadeira, no caso de uma pessoa sentada. Também pode ser pelos pés e pelas costas. A avaliação da vibração é realizada através do método básico de análise usando o valor mais elevado das acelerações ponderadas rms (root meean square) em um dos eixos, expressas em $\mathrm{m} / \mathrm{s}^{2}$ e que são medidas segundo os três eixos ortogonais $\mathrm{x}, \mathrm{y}$ e $\mathrm{z}$ da vibração de translação na superfície que apóia o corpo. $\mathrm{O}$ principal objetivo é avaliar o nível de exposição à vibração em operadores de máquinas empilhadeiras os quais estão expostos no seu dia-a-dia de trabalho e comparar com a Norma Internacional ISO 2631-1 (1997), Mechanical Vibration and Shock - Evalution of Human Exposure to Whole Body Vibration - Part 1: General Requirements. Comparar a amplitude dessa vibração transmitida aos operadores para esse tipo de máquina na direção longitudinal (eixo z), ao longo da coluna lombar, cuja direção influencia diretamente no prejuízo lombar.

\section{Revisão Bibliográfica}

Devido à ampla variedade de possíveis condições e efeitos da exposição humana às vibrações e à escassez de dados seguros, neste trabalho será usada a Norma ISO 2631-1 (1997) (sendo ela inclusive indicada pela Norma Regulamentadora NR-15 (Brasileira), como guia para a avaliação da exposição humana às vibrações de corpo inteiro. Em 2003, o instituto WCB (2003) (Workers' Compensation Board-Engineering Section - órgão sem fins lucrativos do Canadá para a saúde e segurança do trabalhador) foi convidado a participar de um estudo ergonômico para motoristas de ônibus com o 
objetivo de medir e avaliar a vibração nas mãos, transmitida pelo volante (HAV-Hand Arm Vibration) e no corpo inteiro através do assento (WBV-Whole Body Vibration), operando em condições normais. Concluiu-se que os resultados para ambas as medições estavam abaixo do recomendado. Portanto, particularmente, estes motoristas de ônibus não estavam expostos a qualquer risco à saúde, quando relacionado à vibração.

Filho et al (2007) procurou-se determinar os níveis de vibração vertical no assento e na base do assento de um trator agrícola, executando a operação de gradagem para três diferentes velocidades de trabalho. Chegou-se à conclusão que os níveis de aceleração encontrados na parte superior do assento foram, consideravelmente, inferiores aos níveis da base para as três velocidades, sendo a terceira marcha a que apresentou maiores níveis de vibração. No entanto, os valores de aceleração ponderada encontram-se bem acima dos limites definidos na Norma ISO 2631-1 (1997) para um período de 8 horas de trabalho.

Já Brandão (2008) mediu a vibração vertical de empilhadeiras com o objetivo de comparar a vibração no corpo humano de operadores de empilhadeiras, os quais reclamavam que pneus super-elásticos agrediam mais as suas colunas vertebrais. No final, chegou-se à conclusão de que os operadores, quando expostos ao uso de empilhadeiras com pneus tipo pneumático, a vibração era maior e, portanto, mais prejudicial à coluna desses operadores que quando os pneus eram super-elásticos. $\mathrm{O}$ presente trabalho visa, além dos objetivos já expostos, verificar este fato, já que o senso comum indicaria a condição contrária mais perniciosa à saúde. Percebe-se que, da literatura consultada, há controvérsias a respeito se determinado equipamento oferece ou não risco à saúde. De fato, isto irá depender das condições em que as máquinas são operadas, assim como as características técnicas destas máquinas, tempo de exposição e até mesmo características biomédicas das pessoas que operam estas máquinas. Concluise, portanto, que, em cada caso, independente do equipamento ou tempo de exposição, deve-se fazer sempre uma avaliação criteriosa, baseada em números para determinar com precisão os riscos a que estará submetido um determinado trabalhador em uma situação de trabalho na sua jornada diária.

Conhecida no Brasil como "lesões por esforços repetitivos" (LER), a incidência dos distúrbios osteomusculares relacionados ao trabalho (DORT) têm apresentado um crescimento significante e progressivo, nos últimos anos, nas estatísticas oficiais em serviços de atendimento à saúde dos trabalhadores. Apontado como a causa mais freqüente de afastamento do trabalho no mundo, os DORT representam, atualmente, a doença do trabalho mais registrada junto à Previdência Social (Ferreira Junior, 2000).

Em pesquisa realizada a respeito da exposição ocupacional a vibrações e publicada por Melo (1993), é possível constatar a porcentagem ocupada por cada máquina em relação à vibração quando comparada a um todo, conforme é mostrado na Figura 1.

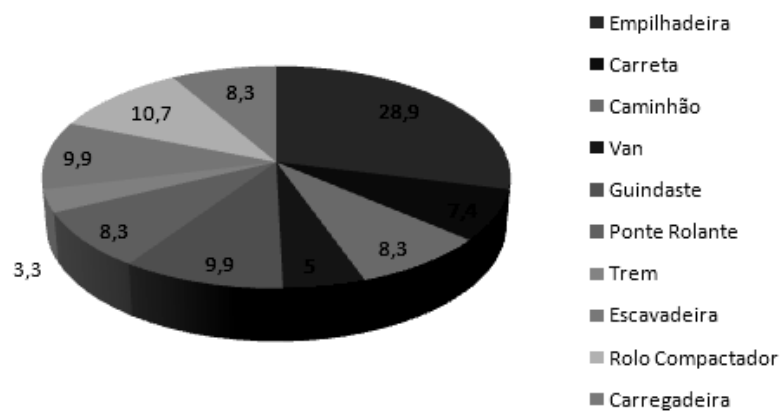

Figura 1: Valor eficaz de aceleração para cada máquina em relação a outras (Les Vibrations aux Postes de Travail, INRS apud Melo, 1993). 
Das máquinas pesquisadas, as quais possuem alguma aplicação industrial, na construção ou serviços em geral, as duas que se destacam pelo alto índice de vibração são as máquinas empilhadeiras e os rolos compactadores. As empilhadeiras são responsáveis por $28,9 \%$ do valor eficaz de aceleração, valor que é responsável direto pela vibração transmitida à pessoa. Enquanto que os rolos compactadores contribuem com $10,7 \%$ do valor total.

\subsection{Avaliação da exposição do corpo humano à vibração}

As vibrações retilíneas transmitidas ao homem devem ser medidas nas direções apropriadas de um sistema coordenado ortogonal, tendo sua origem no centro geométrico da superfície de contato. A terminologia comumente usada na biodinâmica relaciona o sistema coordenado ao esqueleto humano em posição anatômica normal. Acelerações nos pés e nádegas são designadas $\pm \mathrm{a}_{\mathrm{z}}$; acelerações no eixo frente e trás (anteroposterior ou peito/costas), $\pm \mathrm{a}_{\mathrm{x}}$; e no eixo lateral (lado direito para o esquerdo), $\pm \mathrm{a}_{\mathrm{y}}$, conforme mostrado na Figura 2:

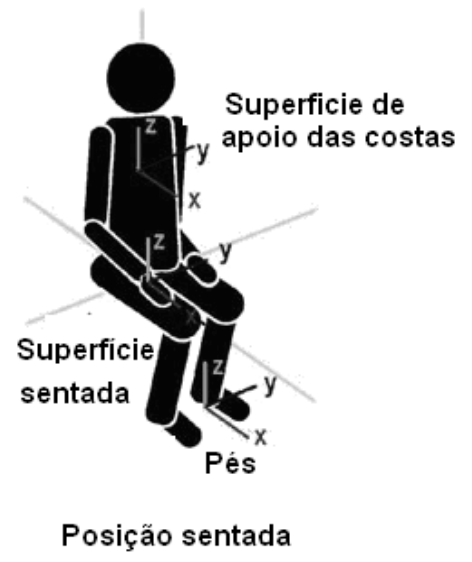

Figura 2: Definição dos eixos ortogonais para medição das acelerações ISSO 2631-1 (1997).

Diferentes fatores de ponderação em freqüências são usados, dependendo da direção de transmissão da vibração ao corpo, como é mostrado nas curvas apresentadas na Figura 3. A idéia destas curvas de ponderação é de funcionar como um filtro, deixando passar para análise apenas a aceleração na freqüência que ele mais prejudica determinada parte do corpo humano. Este trabalho dá ênfase ao problema na saúde, portanto, leva em consideração os fatores de ponderação $W_{k}$, para vibração vertical no eixo z numa faixa que varia entre 4 a $13 \mathrm{~Hz}$ e o fator $W_{d}$ para vibração nos eixos $x$ e $y$ para uma faixa entre 0,5 a $2 \mathrm{~Hz}$. Por se tratar de saúde, também é levado em consideração o fator adicional $W_{c}$. No entanto, este não será abordado, pois as medições foram tomadas somente para o assento e não para o encosto, sem prejuízo algum para os resultados. 


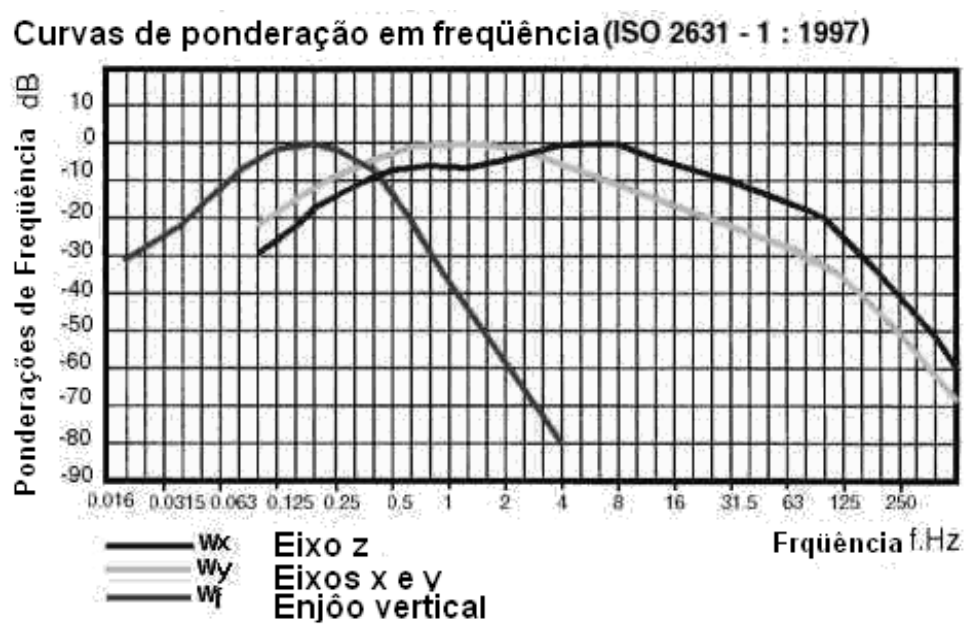

Figura 3: Curvas de ponderação da aceleração em frequiência.

\subsection{Aplicação das ponderações e avaliação do método básico}

O método de avaliação básico da Norma é o método da aceleração ponderada rms. Esse método faz uso da aceleração já ponderada por uma das curvas W, indicadas anteriormente, e é expresso pela Equação (1):

$$
a_{r m s}=\sqrt{\frac{1}{T} \int_{0}^{T} a_{w}^{2}(t) d t}
$$

onde $a_{r m s}$ é a aceleração instantânea $\left(\mathrm{m} / \mathrm{s}^{2}\right) r m s$ já ponderada, T é o tempo de exposição à vibração(s), $a_{w}$ é a aceleração já ponderada pelas curvas de ponderação indicadas anteriormente.

A Norma recomenda outros métodos para o cálculo da aceleração ponderada dependendo da característica da vibração. Para o tipo de vibração transiente, contendo choques de curta duração, quando o "crest factor" (fator de pico) for maior que 9, é recomendado o uso de métodos de avaliação adicionais. O Fator de Crista é a razão entre o valor máximo de aceleração medido dividido pelo valor $r m s$ da aceleração $\left(F=a_{m a x} / a_{r m s}\right)$. Se o "crest factor" for abaixo de 9 , o método de avaliação básico é normalmente suficiente. Caso as acelerações em um dos eixos sejam bem maiores que dos outros dois, a Norma permite que se utilize apenas este valor rms deste eixo de maior vibração. Caso os níveis de vibração rms sejam semelhantes, a Norma recomenda que se utilize a combinação vetorial das três acelerações rms ponderadas vetorialmente, ou seja,

$$
a_{r m s}=\sqrt{k_{x} a_{r m s, x}^{2}+k_{y} a_{r m s, y}^{2}+k_{z} a_{r m s, z}^{2}}
$$

onde $a_{r m s, x}$ é o valor $r m s$ da aceleração $\left(\mathrm{m} / \mathrm{s}^{2}\right)$ no eixo $x$ e $k_{x}$ é um fator multiplicativo que leva em conta a importância da aceleração e o conhecimento estatístico a respeito dos riscos à saúde (utilizando-se a curva $W_{d}$, os fatores são $k_{x}=k_{y}=1,4 \quad e \quad k_{z}=1$, valores estes indicados pela Norma, como as pesquisas a respeito dos efeitos das acelerações ao longo da coluna vertebral é bem estudado, este valor assume $\mathrm{k}_{\mathrm{z}}=1.0$, para as outras direções há valores de majoração, que visam englobar, em caráter preventivo, 
consequiências ainda não observadas ou devidamente investigadas experimentalmente). Outra metodologia, mais sensível a picos de acelerações que o método do valor $r m s$, utiliza uma potência de quarta ordem semelhante ao método para avaliar o valor $r m s$, como indica a Equação (3)

$$
V D V=\sqrt[4]{\int_{0}^{T} a_{w}(t)^{4} d t}
$$

onde VDV é o valor de dose de vibração $\left(\mathrm{m} / \mathrm{s}^{1,75}\right)$ correspondente à aceleração $a_{w}$ instantânea $\left(\mathrm{m} / \mathrm{s}^{2}\right)$ ponderada. No caso de mais de uma exposição à vibração num dia, tem-se que o valor do VDV final é dado por:

$$
V D V=\sum_{i=1}^{n} \sqrt[4]{V D V_{i}^{4}}
$$

onde $n$ é o número de exposições à situações de vibração. Aparentemente, o segundo método poderia ser considerado como mais conservativo já que leva mais em conta valores de pico da aceleração, entretanto curvas limites de vibração diferentes são adotadas neste caso, tornando as duas metodologias praticamente iguais para tempos de exposição de $4 \mathrm{~h}$ a $8 \mathrm{~h}$. Neste trabalho, apenas o primeiro método será utilizado para avaliar o risco da vibração à saúde.

\subsection{Limites de exposição à vibração}

Para a avaliação dos efeitos à saúde, duas relações diferentes podem ser usadas para calcular um valor equivalente de vibração para outro tempo de exposição. Estas equações permitem calcular, dada uma aceleração $r m s$ ponderada $a_{w 1}$ medida durante um determinado tempo $T_{1}$ e avaliar para um tempo $T_{2}$ qual a aceleração equivalente $\mathrm{a}_{\mathrm{w} 2}$ :

$$
\begin{aligned}
& a_{w 1} \sqrt{T_{1}}=a_{w 2} \sqrt{T_{2}} \\
& a_{w 1} \sqrt[4]{T_{1}}=a_{w 2} \sqrt[4]{T_{2}}
\end{aligned}
$$

Cada uma das equações deve ser utilizada em função do modelo de avaliação da vibração (5 no caso do valor $r m s$ da aceleração e 6 no caso do método do VDV da quarta potência). Na Figura 7, apresentam-se as duas equações graficadas. A região entre linhas, para cada uma das equações, representa uma zona de alerta ou cuidado, a qual é indicada por linhas tracejadas na equação (5) e por linhas pontilhadas para a equação (6). Esta região indica que o nível de vibração deve receber atenção, sendo necessário fazer-se algo para que ele seja aliviado. Para a exposição abaixo do limite inferior das equações, o qual é denominado de VAE (Vibration Action Exposure), os efeitos à saúde para os quais as pessoas expostas à vibração estão sujeitos, não são claramente demonstrados e, teoricamente, estão livres de qualquer dano. Dentro da zona, entre os limites inferiores e superiores das equações, existe um risco potencial de que se venha a ter algum problema na saúde. Ao passo que, acima do limite superior da zona, o qual é denominado VLE (Vibration Limit Exposure), o risco de que haja algum problema de saúde é praticamente certo. Percebe-se que, para uma exposição entre $4 \mathrm{~h}$ e 8h, a zona de ação é a mesma para ambas as equações. 


\section{Instrumentação}

Para efetuar as medições, deve ser escolhido um equipamento apropriado, que leve em consideração a localização de captação e o tempo de duração das medições das acelerações. Uma vez que todas as medições estão coletadas, necessitam ser analisadas através de procedimentos adequados e comparadas com a Norma. A vibração é usualmente medida usando acelerômetros alojados de forma ergonômica a não provocar alteração do assento nem mesmo distorcer a postura do operador em excesso. $\mathrm{O}$ transdutor é alocado de forma a seguir o contorno e o formato do banco e contendo um acelerômetro tri-axial para medições simultâneas nos três eixos de vibração.

\subsection{Sistema de medição}

A Norma que define a instrumentação de medição é a ISO 8041(2008) e nela há todos os requerimentos necessários tanto para os procedimentos de instrumentação quanto para os equipamentos e sensores a serem utilizados. Para uma avaliação da exposição completa, existe um requisito para medir todas as características da vibração. Estes equipamentos possuem circuitos ou softwares que permitem fazer a Transformada de Fourier do sinal da aceleração (em escala de decibéis - dB) e assim aplicar as curvas de ponderação, indicadas anteriormente. Dentre as características encontram-se a aceleração em cada freqüência de vibração, a direção da transmissão da vibração ao corpo, o espectro de freqüência e a duração de exposição. Um sistema típico de medição consiste de alguns equipamentos, tais como: Transdutor (acelerômetro + seat-pad) onde são colocados os acelerômetros; amplificador; filtro de frequiência ponderada (permite filtrar a aceleração de entrada para os limites de interesse assim como ponderar estas acelerações de acordo com as freqüências diferentes); armazenador das informações das medidas; analisador de sinal para obter parâmetros relevantes, como aceleração rms, valores de pico, espectros de aceleração, etc.

É importante escolher o acelerômetro de menor massa possível em comparação à massa da pessoa a ser medida. A amplitude dinâmica deve ser capaz de acomodar um nível de aceleração máximo antecipado (não maior que 5G-gravidades no caso de corpo inteiro). A resposta em freqüência deve ser uniforme e constante em toda a faixa de frequiência do espectro medido. $\mathrm{O}$ acelerômetro deve ter uma sensibilidade também adequada aos níveis de vibração que serão medidos, valores da ordem de $100 \mathrm{mV} / \mathrm{g}$ são comuns para este tipo de sensores.

Para a coleta dos dados de aceleração foi utilizado um dosímetro de vibração modelo VI-400, S/N 12430 fabricado pela empresa Quest Technologies (2008), devidamente calibrado. Para configurar, enviar e receber as informações do acelerômetro foi usado o programa Quest Suite PRO VI (2008), o qual oferece a possibilidade de ser pré-ajustado. Antes de qualquer coisa foram selecionados os três canais de medições, $\mathrm{x}, \mathrm{y}$ e $\mathrm{z}$, respectivamente e, dentro desses canais, pode-se optar entre registrar todos os sinais nas medições ou somente o que for de nosso interesse. Aqui, foi selecionado o Buffer " $r m s$ ". Também pode ser ajustada a sensibilidade em cada direção, bem como os modos, as faixas e os tempos de resposta (taxa de registro). No ícone Global, foi possível escolher o tempo de integração, que é o tempo usado como referência para os cálculos de exposição ao grau de vibração, o qual foi usado $8 \mathrm{~h}$. No ícone Avançado, talvez o mais importante a ser configurado, pois apresenta os coeficientes dos vetores " $k$ ", nas três direções e têm influência direta no resultado final dos valores de tempo e, conseqüentemente, nos valores limites de exposição à vibração. Os coeficientes " $\mathrm{K}$ " têm valores diferentes em função do que se deseja analisar. No caso 
de ser relacionado à saúde do operador, os valores são $K_{x}=1,4$ para a aceleração medida em $\mathrm{x}, \mathrm{K}_{\mathrm{y}}=1,4$ para a aceleração medida em y e $\mathrm{K}_{\mathrm{z}}=1,0$ para a aceleração medida em $\mathrm{z}$. Finalizado esse passo, os dados salvos são exportados ao dosímetro e, com isso, pode dar-se início às medições. Nesse estudo, o acelerômetro de sensibilidade $100 \mathrm{mV} / \mathrm{g} \mathrm{em}$ $\mathrm{x} ; 98,3 \mathrm{mV} / \mathrm{g}$ em y e $101,4 \mathrm{mV} / \mathrm{g}$ em z, foi posicionado na interface do assento com o operador, de maneira que os operadores pudessem sentar em cima sem causar qualquer desconforto ou alteração no modo de operar a máquina, tampouco afetasse suas posturas.

\section{Maquinário usado nos ensaios}

Utilizaram-se duas empilhadeiras idênticas, porém com dois tipos diferentes de pneus para a realização das medições. Na figura 4 é mostrada a empilhadeira numa foto tirada de perfil. As máquinas são de marca Clark (2007), modelo CMP25L, capacidade 2,5T, Motor Mitsubishi 4G64, combustível GLP, com deslocados lateral de garfos DLG, Transmissão Clark TA18, torre triplex (3 estágios) 189" e com garfos de $1070 \mathrm{~mm}$. Foram analisadas as acelerações nos três eixos e, posteriormente, calculada a aceleração ponderada, a qual foi confrontada com os dados comparativos da Norma ISO 2631-1. As medições foram realizadas com os operadores sentados, uma vez que a resistência do corpo afeta a transmissão da vibração através do assento. O procedimento de avaliação foi executado em condições normais de trabalho conforme recomendado pela Norma ISO 2631-1. Uma vez executadas as medições, os sinais advindos do acelerômetro foram passados ao programa de aquisição de dados Quest Suite PRO VI, o qual estava instalado em um notebook, através de um cabo USB, onde os dados puderam ser lidos e armazenados.

\subsection{Características dos pneus usados nas empilhadeiras}

Em uma das máquinas foi colocado pneus tipo pneumático e, na outra, pneus super-elásticos ou maciços. A configuração dos pneus empregada em cada máquina é apresentada nas Tabelas 1 e 2, respectivamente:

Tabela 1: Configuração dos pneus para a máquina 1.

\begin{tabular}{|c|c|c|c|c|}
\hline \multirow{2}{*}{ Modelo } & $\begin{array}{c}\text { Especificação } \\
(\mathbf{m m} \times \text { mm) }\end{array}$ & $\begin{array}{c}\text { Especificação } \\
(\mathbf{m m} \text { x mm) }\end{array}$ & Pressão & Pressão \\
\cline { 2 - 5 } & dianteiro & traseiro & dianteiro & traseiro \\
\hline $\begin{array}{c}\text { BKT } \\
\text { PL801 }\end{array}$ & $700 \times 12$ & $650 \times 10$ & 4,8 bar (69 psi) & 5 bar (74 psi) \\
\hline
\end{tabular}

Tabela 2: Configuração dos pneus para a máquina 2.

\begin{tabular}{|c|c|c|c|c|}
\hline \multirow{2}{*}{ Modelo } & Especificação & Especificação & Pressão & Pressão \\
\cline { 2 - 5 } & dianteiro & traseiro & dianteiro & traseiro \\
\hline $\begin{array}{c}\text { Master Solid } \\
\text { Monolitic }\end{array}$ & $700 \times 12$ & $650 \times 10$ & \multicolumn{2}{|c|}{ Maciço } \\
\hline
\end{tabular}




\section{Procedimento de medição com a empilhadeira}

Primeiro foi estabelecido uma simulação real de operação, para que se pudesse coletar os dados mais fiéis possíveis. Escolheram-se quais pisos seriam usados como parâmetros para a realização das operações de medições, sendo o piso 1 de paralelepípedo e o piso 2 de asfalto liso. Cada condição de piso foi realizada com uma empilhadeira com pneus pneumáticos e a outra com super-elásticos. Outra variável foi a condição com ou sem carregamento, usando uma carga comparativa de $1.000 \mathrm{~kg}$. Foi adotada uma sigla para cada configuração de medição, levando em consideração o piso escolhido, a opção de estar com o ou sem carga, o operador a operar a empilhadeira na condição sentado e tipo de pneu utilizado.

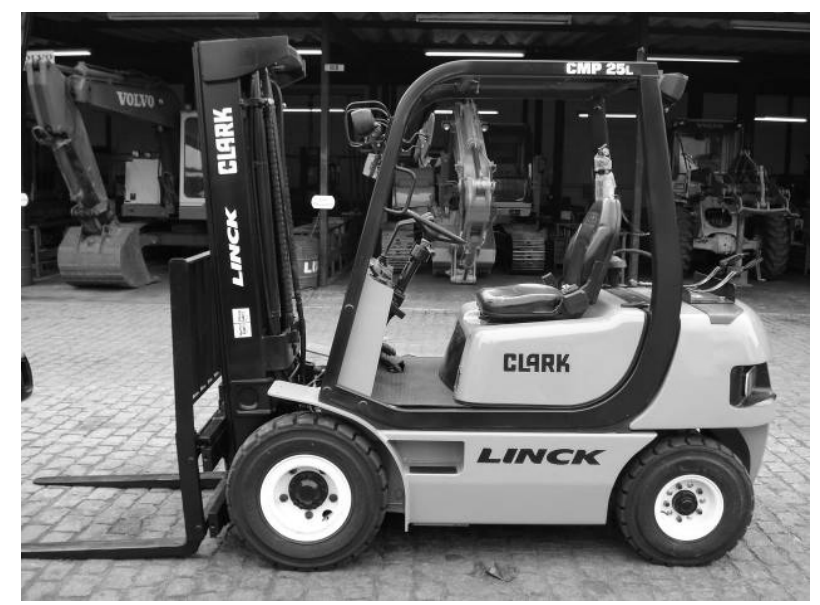

Figura 4: Empilhadeira marca Clark (2007), Modelo CMP25L.

Antes que qualquer medição fosse realizada, foi feita uma entrevista com cada operador, individualmente, solicitando suas opiniões com relação à operação. Ambos reclamaram da operação em piso paralelepípedo na condição sem carregamento e com pneus super-elásticos, alegando ser essa a condição de maior prejuízo para a saúde, o que veio a se confirmar, mais tarde, com a realização dos ensaios. Dois operadores foram selecionados, portanto, para participar do estudo e suas características se encontram na Tabela 3, onde se calculou o Índice de Massa Corpórea de cada um deles $\left(\mathrm{IMC}=\right.$ Massa $\left.(\mathrm{kg}) / \operatorname{Altura}(\mathrm{m})^{2}\right)$ :

Tabela 3: Informações dos operadores que participaram nas medições.

\begin{tabular}{|c|c|c|c|c|}
\hline Operador & $\begin{array}{c}\text { Massa } \\
(\mathbf{k g})\end{array}$ & $\begin{array}{c}\text { Idade } \\
(\mathbf{a n o s})\end{array}$ & $\begin{array}{c}\text { Altura } \\
(\mathbf{m})\end{array}$ & $\begin{array}{c}\text { IMC } \\
\left(\mathbf{k g} / \mathbf{m}^{2}\right)\end{array}$ \\
\hline $\mathbf{A}$ & 75 & 29 & 1,79 & 23,41 \\
\hline $\mathbf{B}$ & 90 & 26 & 1,80 & 27,78 \\
\hline
\end{tabular}

As medições foram executadas de forma a reproduzir a realidade de trabalho. Em função disso, a maneira de operar a máquina e a postura adotada pelo operador foi realizada por eles como se estivessem no dia-a-dia de trabalho sem interferência da medição, como recomenda a boa prática de avaliação de condições insalubres. A coleta foi realizada com um tempo total de aquisição em torno de 60 s. 


\section{Resultados}

Os cálculos feitos para chegar-se à aceleração a qual o operador está submetido a um tempo de exposição de $8 \mathrm{~h}$ foram baseados na equação (5). De forma a ter referências de limites para o prejuízo ou não à exposição à vibração são usados dois valores limites. Aplicando-se as equações anteriormente mostradas da ISO 2631-1, para o limite inferior é usado $\mathrm{VAE}=0,5045 \mathrm{~m} / \mathrm{s}^{2}$ e para o limite superior é usado VLE= $0,8291 \mathrm{~m} / \mathrm{s}^{2}$ para um tempo de exposição de $\mathrm{T}=8 \mathrm{~h}$.

Conforme mostrado na Figura 5, as acelerações captadas na operação, onde o operador está exposto a uma condição com um piso de paralelepípedo e com pneus super-elásticos, são relativamente maiores quando comparadas às acelerações com pneus pneumáticos.

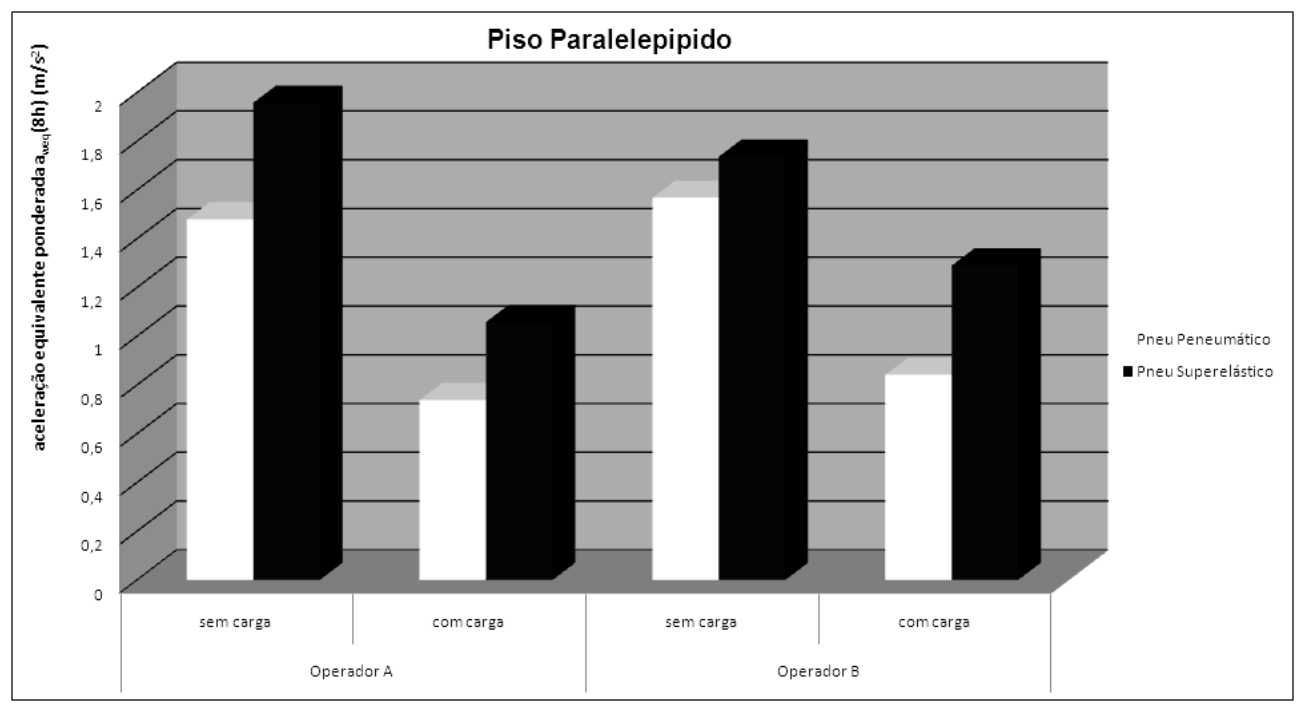

Figura 5: Nível de aceleração ponderada no assento estendido para 8h sob diversas condições de carga e operadores (Condição Piso de Paralelepípedo).

Na condição 'sem carregamento', a mais prejudicial de todas as situações, para ambos os operadores, a vibração afeta, de maneira considerável (VLE - Valor limite de Exposição) a coluna do operador, quando esse fica exposto a essas condições por mais de $1 \mathrm{~h}$ e $24 \mathrm{~min}$ para o operador A e $1 \mathrm{~h}$ e $47 \mathrm{~min}$ para o operador B, como pode ser comprovado nos valores registrados na Tabela 4.

Tabela 4: Piso com paralelepípedo e com pneus tipo super-elástico.

\begin{tabular}{|c|c|c|c|c|c|c|c|c|}
\hline Medição & Operador & Carga & $\begin{array}{c}\mathbf{a}_{\mathbf{x}} \\
\left(\mathbf{m} / \mathbf{s}^{\mathbf{2}}\right)\end{array}$ & $\begin{array}{c}\mathbf{a}_{\mathbf{y}} \\
\left(\mathbf{m} / \mathbf{s}^{\mathbf{2}}\right)\end{array}$ & $\begin{array}{c}\mathbf{a}_{\mathbf{z}} \\
\left(\mathbf{m} / \mathbf{s}^{\mathbf{2}}\right)\end{array}$ & $\begin{array}{c}\boldsymbol{a}_{\text {rms }} \\
\left(\mathbf{m} / \mathbf{s}^{\mathbf{2}}\right)\end{array}$ & $\begin{array}{c}\text { Tempo } \\
\mathbf{V A E} \\
\mathbf{h h : m m}\end{array}$ & $\begin{array}{c}\text { Tempo } \\
\mathbf{V L E} \\
\mathbf{h h : m m}\end{array}$ \\
\hline $\mathbf{1}$ & $\mathrm{A}$ & sem & 0,6202 & 0,4814 & 1,6199 & 1,9576 & $00: 31$ & $01: 24$ \\
\hline $\mathbf{2}$ & $\mathrm{B}$ & $\mathrm{sem}$ & 0,6102 & 0,6599 & 1,1967 & 1,7366 & $00: 39$ & $01: 47$ \\
\hline $\mathbf{3}$ & $\mathrm{B}$ & $\mathrm{com}$ & 0,4217 & 0,4227 & 0,9817 & 1,2894 & $01: 12$ & $03: 14$ \\
\hline $\mathbf{4}$ & A & com & 0,378 & 0,3728 & 0,7534 & 1,0583 & $01: 47$ & $04: 48$ \\
\hline
\end{tabular}

Ao passo que, na condição 'com carregamento', o trabalhador pode ficar um pouco mais de tempo exposto a tal condição. O operador A terá um prejuízo (VLE - 
Valor limite de Exposição) a partir de 4h e 48min de trabalho, enquanto que o operador B, com 3 h e 14min. Esses tempos de exposição são considerados baixo, quando comparados a uma jornada de trabalho padrão de $8 \mathrm{~h}$.

$\mathrm{Na}$ segunda situação, apresentada na Figura 6, o operador está exposto a uma condição de trabalho onde o piso é de asfalto liso e com pneus pneumáticos, caracterizase em um menor prejuízo à saúde do operador em relação ao piso de paralelepípedo. $\mathrm{Na}$ condição menos prejudicial, "com carregamento", os operadores teriam a saúde afetada com mais de $24 \mathrm{~h}$ de exposição.

Já na condição "sem carregamento", o operador A, com $15 \mathrm{~h}$ e $32 \mathrm{~min}$ sofreria o desgaste provocado pela vibração; enquanto que o B, com $16 \mathrm{~h}$ e $27 \mathrm{~min}$ de exposição, como pode ser conferido na Tabela 5. Portanto, quando comparadas às duas condições, fica evidente que a operação em piso asfaltado, com pneus pneumáticos e com carregamento é menos prejudicial à saúde do operador e, com isso, há possibilidade de tirar o máximo rendimento do trabalhador.

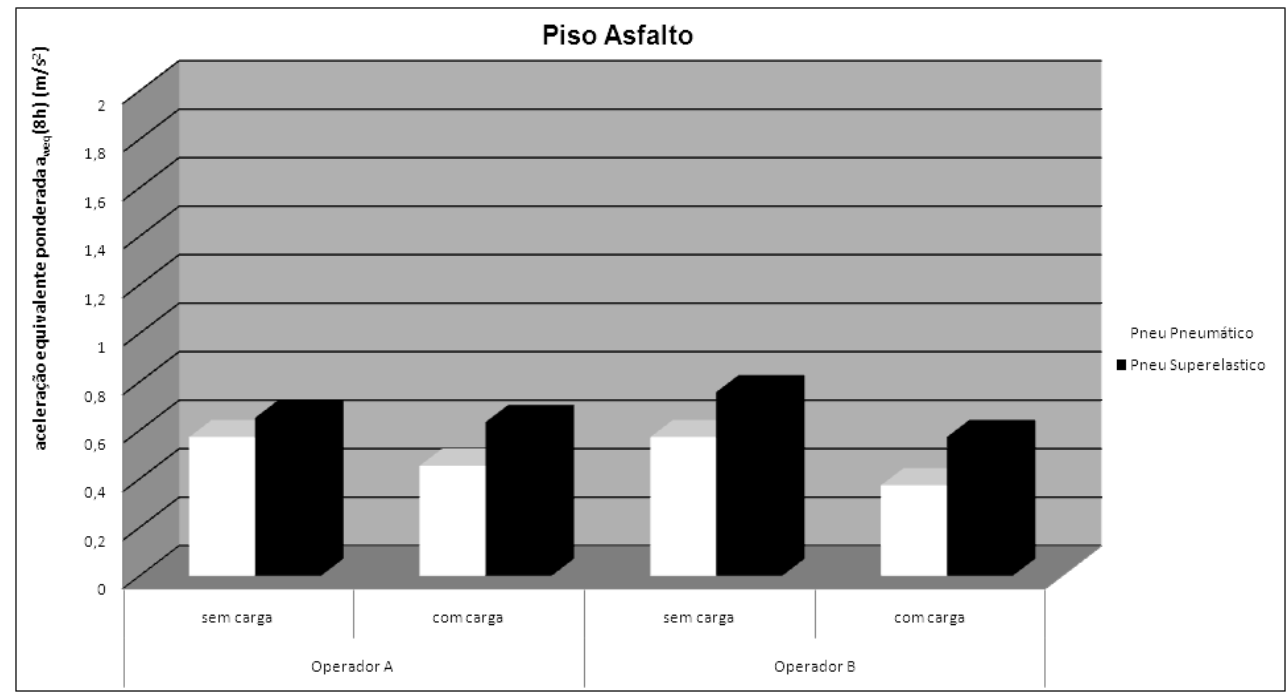

Figura 6: Nível de aceleração ponderada no assento estendido para 8h sob diversas condições de carga e operadores (Condição Piso de Asfalto).

Tabela 5: Piso com asfalto liso e com pneus tipo pneumático.

\begin{tabular}{|c|c|c|c|c|c|c|c|c|}
\hline Medição & Operador & Carga & $\begin{array}{c}\mathbf{a}_{\mathbf{x}} \\
\left(\mathbf{m} / \mathbf{s}^{\mathbf{2}}\right)\end{array}$ & $\begin{array}{c}\mathbf{a}_{\mathbf{y}} \\
\left(\mathbf{m} / \mathbf{s}^{2}\right)\end{array}$ & $\begin{array}{c}\mathbf{a}_{\mathbf{z}} \\
\left(\mathbf{m} / \mathbf{s}^{\mathbf{2}}\right)\end{array}$ & $\begin{array}{c}\boldsymbol{a}_{\text {rms }} \\
\left(\mathbf{m} / \mathbf{s}^{\mathbf{2}}\right)\end{array}$ & $\begin{array}{c}\text { Tempo } \\
\mathbf{V A E} \\
\mathbf{h h : m m}\end{array}$ & $\begin{array}{c}\text { Tempo } \\
\mathbf{V L E} \\
\mathbf{h h}: \mathbf{m m}\end{array}$ \\
\hline $\mathbf{5}$ & $\mathrm{B}$ & com & 0,1669 & 0,1545 & 0,1941 & 0,3729 & $14: 22$ & $>24$ \\
\hline $\mathbf{6}$ & $\mathrm{A}$ & com & 0,165 & 0,2339 & 0,2133 & 0,454 & $09: 42$ & $>24$ \\
\hline $\mathbf{7}$ & $\mathrm{B}$ & sem & 0,1892 & 0,2732 & 0,3323 & 0,5718 & $06: 07$ & $16: 27$ \\
\hline $\mathbf{8}$ & A & sem & 0,1732 & 0,2822 & 0,3474 & 0,5824 & $05: 54$ & $15: 32$ \\
\hline
\end{tabular}

Outros resultados extraídos dos ensaios são apresentados a seguir nas Tabelas 6 e 7 podem ser usados para análises comparativas.

Tabela 6: Piso com paralelepípedo e com pneus tipo pneumático.

\begin{tabular}{|c|c|c|c|c|c|c|c|c|}
\hline Medição & Operador & Carga & $\begin{array}{c}\mathbf{a}_{\mathbf{x}} \\
\left(\mathbf{m} / \mathbf{s}^{2}\right)\end{array}$ & $\begin{array}{c}\mathbf{a}_{\mathbf{y}} \\
\left(\mathbf{m} / \mathbf{s}^{2}\right)\end{array}$ & $\begin{array}{c}\mathbf{a}_{\mathbf{z}} \\
\left(\mathbf{m} / \mathbf{s}^{2}\right)\end{array}$ & $\begin{array}{c}\boldsymbol{a}_{r m s} \\
\left(\mathbf{m} / \mathbf{s}^{2}\right)\end{array}$ & $\begin{array}{c}\text { Tempo } \\
\text { VAE } \\
\mathbf{h h : m m}\end{array}$ & $\begin{array}{c}\text { Tempo } \\
\mathbf{V L E} \\
\mathbf{h h m}\end{array}$ \\
\hline
\end{tabular}




\begin{tabular}{|c|c|c|c|c|c|c|c|c|}
\hline $\mathbf{9}$ & A & sem & 0,4421 & 0,5041 & 1,1429 & 1,4789 & $00: 54$ & $02: 27$ \\
\hline $\mathbf{1 0}$ & B & sem & 0,5761 & 0,6159 & 1,0316 & 1,5678 & $00: 48$ & $02: 11$ \\
\hline $\mathbf{1 1}$ & B & com & 0,3436 & 0,3323 & 0,5099 & 0,8413 & $02: 49$ & $07: 36$ \\
\hline $\mathbf{1 2}$ & A & com & 0,2591 & 0,2894 & 0,4989 & 0,738 & $03: 40$ & $09: 52$ \\
\hline
\end{tabular}

Tabela 7: Piso com asfalto liso e com pneus tipo super-elástico.

\begin{tabular}{|c|c|c|c|c|c|c|c|c|}
\hline Medição & Operador & Carga & $\begin{array}{c}\mathbf{a}_{\mathbf{x}} \\
\left(\mathbf{m} / \mathbf{s}^{\mathbf{2}}\right)\end{array}$ & $\begin{array}{c}\mathbf{a}_{\mathbf{y}} \\
\left(\mathbf{m} / \mathbf{s}^{2}\right)\end{array}$ & $\begin{array}{c}\mathbf{a}_{\mathbf{z}} \\
\left(\mathbf{m} / \mathbf{s}^{\mathbf{2}}\right)\end{array}$ & $\begin{array}{c}\boldsymbol{a}_{\text {rms }} \\
\left(\mathbf{m} / \mathbf{s}^{\mathbf{2}}\right)\end{array}$ & $\begin{array}{c}\text { Tempo } \\
\mathbf{V A E} \\
\mathbf{h h : m m}\end{array}$ & $\begin{array}{c}\text { Tempo } \\
\mathbf{V L E} \\
\mathbf{h h}: \mathbf{m m}\end{array}$ \\
\hline $\mathbf{1 3}$ & $\mathrm{B}$ & com & 0,2048 & 0,1965 & 0,3737 & 0,5715 & $04: 07$ & $09: 17$ \\
\hline $\mathbf{1 4}$ & $\mathrm{A}$ & $\mathrm{com}$ & 0,2003 & 0,2609 & 0,3221 & 0,6334 & $05: 00$ & $10: 24$ \\
\hline $\mathbf{1 5}$ & $\mathrm{B}$ & sem & 0,2407 & 0,2927 & 0,3855 & 0,7569 & $06: 40$ & $11: 30$ \\
\hline $\mathbf{1 6}$ & $\mathrm{A}$ & sem & 0,2654 & 0,3013 & 0,4710 & 0,651 & $08: 16$ & $11: 33$ \\
\hline
\end{tabular}

\section{Análise dos Resultados e Conclusões}

A partir da análise dos resultados, chegou-se a conclusão que os operadores de empilhadeiras estão expostos a valores de vibração maiores do que são recomendados pela Norma ISO 2631, para a maioria dos casos, para uma jornada de trabalho de 8 horas. Percebe-se que de todas as medições feitas, 43,5\% se encontram acima da VLE, condição considerada prejudicial com elevado grau de certeza. As medições situadas na faixa entre a VAE e VLE, zona considerada potencialmente prejudicial, onde existe o risco de que o operador exposto venha a se prejudicar com a operação, representam 44\%. Os valores que estão abaixo do limite VAE da curva da equação B1, onde, teoricamente, o operador está livre de qualquer prejuízo à sua saúde, representam apenas $12,5 \%$ das medições, como pode ser visto na Figura 7.

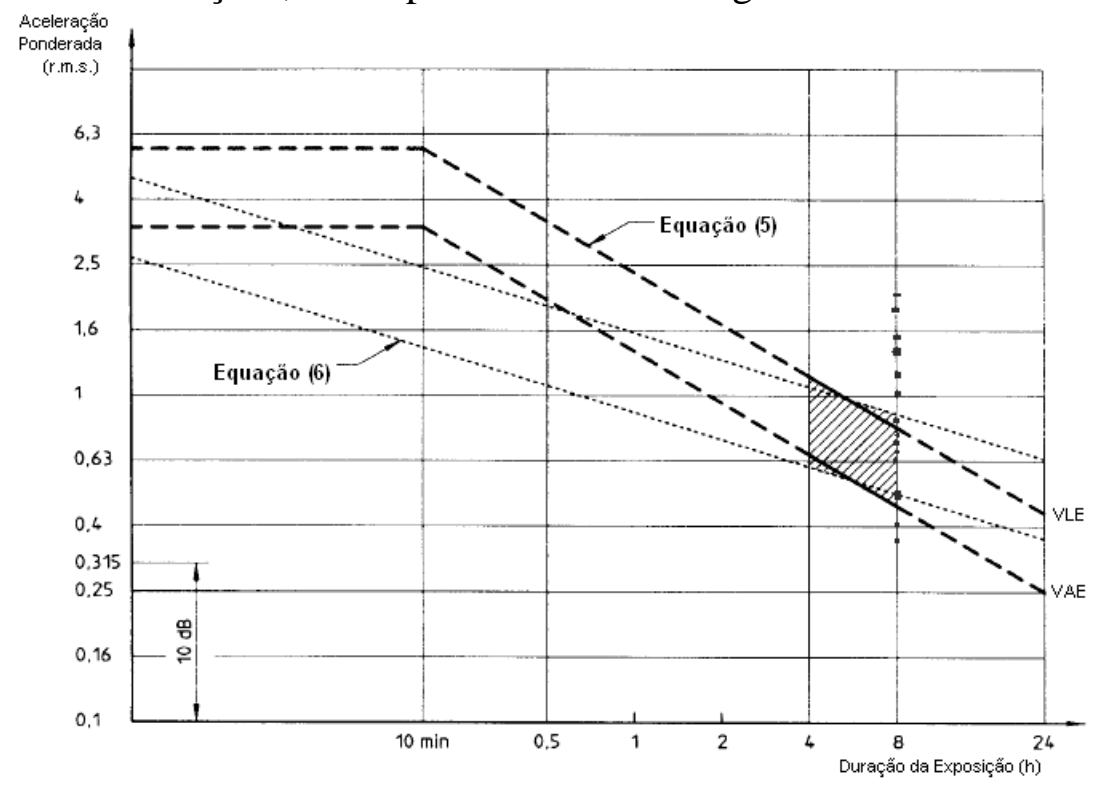

Figura 7: Acelerações ponderadas no assento estendido para $8 \mathrm{~h}$ sob diversas condições de carga e operadores.

A segunda conclusão que fica evidente é que a operação, na condição com pneus super-elásticos, apresenta valores para a aceleração maiores que com pneus 
pneumáticos, em qualquer piso que a máquina esteja trabalhando. Isso se dá pelo fato de grande parte do choque absorvido pelo pneu maciço ser transmitido ao operador, ao passo que os pneumáticos, mesmo que insignificante, fazem o papel de amortecedor. Uma terceira constatação é que, na condição de operação com carregamento, a vibração é menor. Isso se explica pelo fato da empilhadeira ter o passeio do seu centro de gravidade (CG) situado dentro de um triângulo chamado de Zona de Estabilidade da empilhadeira. À medida que se aumenta a carga de carregamento, o CG desloca para frente, ficando em condição de maior estabilidade. Caso contrário, desloca-se para trás, situando-se na parte mais instável e assim, vibrando mais. Apesar de apresentar algumas desvantagens em relação ao pneu super-elástico, como a necessidade de calibração constante, o fato de furar com facilidade e desgaste em tempo menor, o pneu do tipo pneumático oferece maiores benefícios no quesito saúde e conforto no momento da operação e, em função disso, deve ser levado em consideração na hora da escolha.

\section{Referências bibliográficas}

Brandão, J.G.T.; Tomazini, J.E.; Amann B.O. (2008), Análise de Vibrações em assentos de empilhadeira. 7th Brazilian Conference on Dynamics, Control and Applications. Dincon.

Clark, (2007), Catálogo Informativo das Empilhadeiras Clark.

http://www.clarkempilhadeiras.com.br, Acessado em 12/ de Setembro de 2008.

Ferreira Junior, M. , (2000), Saúde no trabalho: temas básicos para o profissional que cuida da saúde dos trabalhadores. São Paulo: Roca, 357p.

Filho, P. F.; Fernandes, H. C.; Queiroz, D. M.; Souza, A. P.; Camilo, A. J., (2007), Avaliação dos Níveis de Vibração Vertical no Assento de um Trator Agrícola de Pneus Utilizando um Sistema de Aquisição Automática de Dados, Revista Árvore, Viçosa-MG, v.27, n.6, p.887-895.

Melo, R., (1993), Health and Safety Executive, United Kingdom, 58p.

ISO 2631-1, (1997), Mechanical Vibration and Shock - Evalution of Human Exposure to Whole Body Vibration - Part 1: General Requirements.

ISO 5349-1 (1997), Mechanical vibration - Measurement and evaluation of human exposure to hand-transmitted vibration - Part 1: General requirements.

ISO 5349-2, (1997), Mechanical vibration - Measurement and evaluation of human exposure to hand-transmitted vibration - Part 2: Practical Guidance for measurement at the workplace.

ISO 8041, (2008) - Human response to vibration - Measuring instrumentation, http://www.techstreet.com/cgi-bin/detail?product_id=32527. Acessado em $10 \mathrm{de}$ Setembro.

Quest-Technology, (2008), Vibration monitors and Real-time vibration Analyzers, VI400 PRO, 203p.

Palmer, K. T., Griffin, M. J., Bendall, H., Pennett, B. (2000), Prevalence and pattern of occupational exposure to whole-body vibration in Great Britain: findings from a national survey. Occupational Environmental Medicine, p.229-236.

NR 15, (2008), Norma Regulamentadora do Ministério do Trabalho e Emprego, Anexo 8, Brasil, http://www.mte.gov.br/legislacao/normas_regulamentadoras/nr15.pdf Acessado em 10 de Setembro de 2008.

WCB Workers Compensation Board of BC, (2003), Bus Drivers \& Human Vibration, Engineering Section Report, Vancouver, Canada, ARCS Reference No: 0135-20, 10p.,Sep. 


\title{
MEASUREMENT AND EVALUATION OF VIBRATION LEVELS ON FORKLIFT TRUCK DRIVERS
}

\begin{abstract}
Extended Abstract
This paper shows a whole-body vibration study in forklift trucks operators working in different situations, comparing two kinds of tires, pneumatic and super elastic (massive); two kinds of floor, regular paving stone and smooth asphalt, and two load conditions, load-free or full loaded in equivalent speeds. The vibration was measured in three orthogonal axes, $\mathrm{x}, \mathrm{y}$ and $\mathrm{z}$, and then calculated a weighted root-mean-square acceleration to compare the results with the ISO 2631-1 (1997) standard. The main goal of this paper was to compare the human whole-body vibration in forklift trucks operators, who are exposed to such repetitive load and complain about discomfort on their spines during normal machine operation. In general, it was possible to verify that the use of pneumatic tires resulted in lower values of equivalent acceleration. In this case, the super elastic tires showed results with higher acceleration magnitude.

It is usual to separate two main vibration source categories for human beings: those that involves whole body vibration and those that involves just some human limbs, such as, hand-arm segment. The transmitted vibrations are generally low frequency, high amplitude accelerations. They are in the 1 to $80 \mathrm{~Hz}$ frequency range, specifically in the 1 to $20 \mathrm{~Hz}$ range. The motion sickness is also treated as wholly body vibration and it acts in the frequency range of 0.1 to $0.63 \mathrm{~Hz}$. Such vibrations are more critical to activities related to transportations systems. Vibrations that reach the hand-arm segment (localized vibrations) are frequently studied and occur in the 6.3 to $1250 \mathrm{~Hz}$ range. Filho et al. (2003) presented a paper where the vertical vibrations on agricultural tractors in the seat's driver, operating with plow and three service speeds. It was concluded that the acceleration levels on the seat were considerable lower than the levels measured on the base of the seat for the three investigated speeds, and the third gear presented the higher levels of vibration. However, the weighted acceleration levels were beyond ISO 2631-1 (1997) Standard limits for a work day (8 hours). Brandão (2008) measured the vertical vibration on forklift trucks aiming at the comparisons for truck driver vibrations who complained about the use of super elastic tires (they frequently generated lumbar column fatigue). Finally they concluded that the truck drivers when exposed to vibrations generated by pneumatic tires equipped trucks were exposed to higher level of vibration and therefore this condition were more harmful to health compared to super elastic tires. It was noticed that for the consulted literature there are some controversies if some equipments presents or not risk to human health. In fact, this will depend on operational conditions and technical characteristics of these machines as well. Another important item is related to the exposure to vibration, driver's biometric characteristics. It was concluded that in each case, independently of the used equipment or the exposure time a critic appraisal should be done, based on the measurements, in order to precisely evaluate the risks to vibrations.

The used vehicles in the tests were identical. Figure 1 shows the forklift used in the tests. The vehicle presents the following features. Forklift by Clark, model CMP25L, 2,5 ton capacity, Mitsubishi 4G64 engine, GLP fuel, with forks and retracted side
\end{abstract}


supports, DLG, Clark transmission TA18, triplex turret (3 stages) 189" and forks with $1070 \mathrm{~mm}$. The computations were made aiming the equivalent acceleration for $8 \mathrm{~h}$ daily work journey and using the second equation indicated by ISO Standard. In order to have limit references for harmful vibration it is used two limit values (vibration action limit and vibration limit exposure). Using the previously equations indicated by ISO 2631-1, the lower limit used was $\mathrm{VAE}=0.5045 \mathrm{~m} / \mathrm{s}^{2}$ and the upper limit used was VLE $=0.8291$ $\mathrm{m} / \mathrm{s}^{2}$ for $8 \mathrm{~h}$ exposure time.

As indicated by Table 1, the acquired vibrations for block pavement and solid tires are relatively higher than those with pneumatic tires. In the unload condition, the most harmful condition for both drivers, the vibration affects considerably driver column (VLE) if driver A exposes to more than $1 \mathrm{~h} 24 \mathrm{~min}$ and for driver B more than $1 \mathrm{~h} 47 \mathrm{~min}$, as indicated by Table 1 . On the other hand, for load condition operation, the driver might be exposed to longer times. Driver A will have harmful effects(VLE) with exposure times more than $4 \mathrm{~h} 48 \mathrm{~min}$ while driver B has a limit of $3 \mathrm{~h} 14 \mathrm{~min}$ of exposure. These exposure times are considered low values when compared with a daily $8 \mathrm{~h}$ work journey. The second situation referrers to smooth asphalt pavement and pneumatic tires (Table 2). This condition showed less harmful to healthy than the previous situation (block pavement). In this case, the less harmful situation, loaded, the drivers would be exposed for such vibrations for more than $24 \mathrm{~h}$. However, in the unloaded condition, driver A would be exposed to a limit of $15 \mathrm{~h} 32 \mathrm{~min}$, while driver B would be exposed to a limit of $16 \mathrm{~h} 27 \mathrm{~min}$ as can be noticed in the Table 2 . Therefore, when the two conditions are faced, it is evident that the smooth asphalt pavement with pneumatic tires and loaded presents less harmful vibrations to driver healthy and thus the driver would not suffer injuries and would work the full journey without injuries.

Analyzing the results, it was concluded that some forklift drivers in certain conditions are exposed to vibration values above ISO 2631 Standard limits recommendations (in this work, most of the cases) for a daily $8 \mathrm{~h}$ work journey. It can be noticed that $43.5 \%$ of measurements are above VLE limit, which is a harmful condition with high level of probability of injury. Measurements between VAE and VLE limits, which is a zone considered potentially harmful, represents $44 \%$ of the measurements. $12.5 \%$ of the measurements presented equivalent measurements VAE limit for the second equation and theoretically, drivers subjected to such vibrations will not present any healthy effects during his work life. The second remarkable conclusion is that the operation with solid tires presents values of vibration higher than pneumatic tires for any type of pavement. This probably is due to the fact that impact absorbed by the tire is largely transmitted to the driver since here is no suspension system. On the other hand, the pneumatic tires absorb part of the vibration as a dashpot. At last but not at least is that in the loaded condition the vibrations are less intense. This can be explained by the fact that forklift center of gravity (CG) shifts accordingly with the load in the fork.

Table 1. Measurements on block pavement and solid tires.

\begin{tabular}{|c|c|c|c|c|c|c|c|c|}
\hline Measurement & Driver & Load & $\begin{array}{c}\mathbf{a}_{\mathbf{x}} \\
\left(\mathbf{m} / \mathbf{s}^{2}\right)\end{array}$ & $\begin{array}{c}\mathbf{a}_{\mathbf{y}} \\
\left(\mathbf{m} / \mathbf{s}^{\mathbf{2}}\right)\end{array}$ & $\begin{array}{c}\mathbf{a}_{\mathbf{z}} \\
\left(\mathbf{m} / \mathbf{s}^{\mathbf{2}}\right)\end{array}$ & $\begin{array}{c}\boldsymbol{a}_{\text {rms }} \\
\left(\mathbf{m} / \mathbf{s}^{\mathbf{2}}\right)\end{array}$ & $\begin{array}{c}\text { Tempo } \\
\mathbf{V A E} \\
\mathbf{h h : m m}\end{array}$ & $\begin{array}{c}\text { Tempo } \\
\mathbf{V L E} \\
\mathbf{h h}: \mathbf{m m}\end{array}$ \\
\hline $\mathbf{1}$ & A & without & 0.6202 & 0.4814 & 1.6199 & 1.9576 & $00: 31$ & $01: 24$ \\
\hline $\mathbf{2}$ & B & without & 0.6102 & 0.6599 & 1.1967 & 1.7366 & $00: 39$ & $01: 47$ \\
\hline $\mathbf{3}$ & B & with & 0.4217 & 0.4227 & 0.9817 & 1.2894 & $01: 12$ & $03: 14$ \\
\hline $\mathbf{4}$ & A & with & 0.378 & 0.3728 & 0.7534 & 1.0583 & $01: 47$ & $04: 48$ \\
\hline
\end{tabular}


Table 2. Measurements on smooth asphalt pavement and pneumatic tires.

\begin{tabular}{|c|c|c|c|c|c|c|c|c|}
\hline Measurement & Driver & Load & $\begin{array}{c}\mathbf{a}_{\mathbf{x}} \\
\left(\mathbf{m} / \mathbf{s}^{2}\right)\end{array}$ & $\begin{array}{c}\mathbf{a}_{\mathbf{y}} \\
\left(\mathbf{m} / \mathbf{s}^{\mathbf{2}}\right)\end{array}$ & $\begin{array}{c}\mathbf{a}_{\mathbf{z}} \\
\left(\mathbf{m} / \mathbf{s}^{2}\right)\end{array}$ & $\begin{array}{c}\boldsymbol{a}_{\text {rms }} \\
\left(\mathbf{m} / \mathbf{s}^{\mathbf{2}}\right)\end{array}$ & $\begin{array}{c}\text { Tempo } \\
\mathbf{V A E} \\
\mathbf{h h : m m}\end{array}$ & $\begin{array}{c}\text { Tempo } \\
\mathbf{V L E} \\
\mathbf{h h}: \mathbf{m m}\end{array}$ \\
\hline $\mathbf{5}$ & B & with & 0.1669 & 0.1545 & 0.1941 & 0.3729 & $14: 22$ & $>24$ \\
\hline $\mathbf{6}$ & A & with & 0.165 & 0.2339 & 0.2133 & 0.454 & $09: 42$ & $>24$ \\
\hline $\mathbf{7}$ & B & without & 0.1892 & 0.2732 & 0.3323 & 0.5718 & $06: 07$ & $16: 27$ \\
\hline $\mathbf{8}$ & A & without & 0.1732 & 0.2822 & 0.3474 & 0.5824 & $05: 54$ & $15: 32$ \\
\hline
\end{tabular}

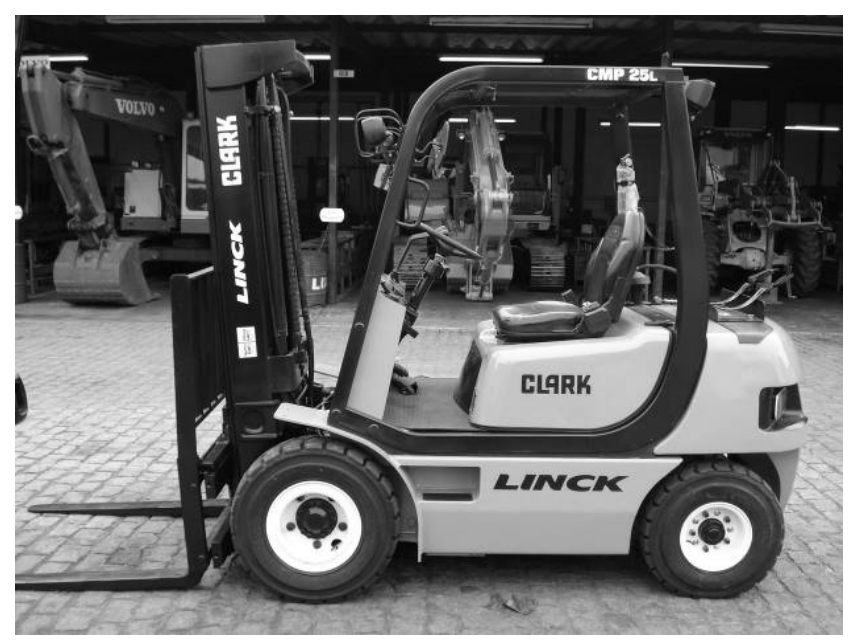

Figure 1. Clark Forklift Model CMP25L. 